\title{
Effects of Attention on Orientation-Tuning Functions of Single Neurons in Macaque Cortical Area V4
}

\author{
Carrie J. McAdams ${ }^{1}$ and John H. R. Maunsell ${ }^{1,2}$ \\ ${ }^{1}$ Division of Neuroscience and ${ }^{2 H}$ Howard Hughes Medical Institute, Baylor College of Medicine, Houston, Texas 77030
}

\begin{abstract}
We examined how attention affected the orientation tuning of 262 isolated neurons in extrastriate area V4 and 135 neurons in area V1 of two rhesus monkeys. The animals were trained to perform a delayed match-to-sample task in which oriented stimuli were presented in the receptive field of the neuron being recorded. On some trials the animals were instructed to pay attention to those stimuli, and on other trials they were instructed to pay attention to other stimuli outside the receptive field. In this way, orientation-tuning curves could be constructed from neuronal responses collected in two behavioral states: one in which those stimuli were attended by the animal and one in which those stimuli were ignored by the animal. We
\end{abstract}

The responses of neurons in extrastriate visual cortex are often enhanced when an animal must attend to or remember a stimulus in the receptive field (Desimone and Duncan, 1995; Maunsell, 1995). Attentional enhancement has been found in many different visual areas, including areas relatively early in visual processing and in later stages of both the parietal and temporal pathways. Although these modulations are sometimes modest, their strength has been shown to depend on the demands of the tasks (Spitzer et al., 1988; Luck et al., 1997), and in some conditions robust effects are seen (Moran and Desimone, 1985; Motter, 1994a; Treue and Maunsell, 1996).

These enhanced neural responses could account for the improved behavioral performance associated with attention. Attending to a particular object, location, or feature can improve detection thresholds and speed behavioral responses (Posner et al., 1980; Humphreys and Bruce, 1989; Rossi and Paradiso, 1995). Stronger neuronal responses typically have a better signal-tonoise ratio (Schiller et al., 1976; Heggelund and Albus, 1978; Geisler and Albrecht, 1997), which might provide the basis for superior behavioral responses. It is possible, however, that changes in behavior performance depend on other neurophysiological effects of attention. If attention altered the stimulus selectivities of individual neurons, those cells could signal attributes of the attended stimulus more precisely. A sharpening of tuning curves for an attended stimulus dimension, such as orientation or color, could provide a finer-grained representation that would

\footnotetext{
Received June 17, 1998; revised Oct. 15, 1998; accepted Oct. 20, 1998.

This work was supported by the National Institutes of Health (Grants RO1 EY05911, T32 EY07001, T32 GM07330, and T32 GM08507 to C.J.M.). J.H.R.M. is a Howard Hughes Medical Institute Investigator. We thank D. Sparks, E. Cook, C. E. Boudreau, and G. Ghose for helpful comments on preliminary versions of this manuscript. We also thank C. E. Boudreau, R. Diaz, and B. Noerager for help with training the animals and other technical assistance.

Correspondence should be addressed to Carrie J. McAdams, Division of Neuroscience, Baylor College of Medicine, One Baylor Plaza, Houston, TX 77030.

Copyright (C) 1998 Society for Neuroscience 0270-6474/98/190431-11\$05.00/0
}

fit Gaussians to the neuronal responses to twelve different orientations for each behavioral state. Although attention enhanced the responses of V4 neurons (median 26\% increase) and $\mathrm{V} 1$ neurons (median $8 \%$ increase), selectivity, as measured by the width of its orientation-tuning curve, was not systematically altered by attention. The effects of attention were consistent with a multiplicative scaling of the driven response to all orientations. We also found that attention did not cause systematic changes in the undriven activity of the neurons.

Key words: attention; visual; orientation; tuning; monkey; area V4; extrastriate; cortex improve the animal's performance in reporting information about the stimulus.

The question of whether attention can alter tuning curves has been considered in earlier studies (Haenny and Schiller, 1988; Spitzer et al., 1988), which suggested that attention to oriented stimuli causes a systematic narrowing of orientation-tuning curves in area V4. On the other hand, some studies of extraretinal inputs to cortex have either failed to find evidence of narrowing of spatial or feature-directed tuning profiles (Andersen et al., 1985; Vogels and Orban, 1990) or were inconclusive (Maunsell and Hochstein, 1991). We were motivated to reexamine this issue because of evidence suggesting that the narrowing of tuning curves is uncommon in stimulus-stimulus interactions. For example, increasing the contrast of a visual stimulus typically increases responses without altering the selectivity of a cell for orientation or spatial frequency (Tolhurst, 1973; Dean, 1981; Albrecht and Hamilton, 1982; Sclar and Freeman, 1982). Thus, if attention does alter the tuning of neurons, it would support the notion that attentional inputs to cortex operate through mechanisms or pathways that are distinct from those primarily involved with sensory signals.

\section{MATERIALS AND METHODS}

Behavioral paradigms. We examined the effects of attention on the orientation tuning and undriven activity of single neurons in cortical area V4 by recording from neurons in monkeys while they performed a task that required them to shift their attention between two stimulus locations. Data were collected from two male rhesus monkeys (Macaca mulatta). Each sat in a primate chair during training and recording sessions, which lasted 2-6 hr, and was unrestrained in its home cage at other times. Water intake was controlled, and each animal was trained to perform a behavioral task using operant conditioning with a juice reward. In all cases the animal was required to maintain fixation on a small spot so that visual stimuli could be placed at known retinal locations. Partway through training, an aseptic surgery was performed to implant a head post and scleral search coil (Judge et al., 1980), which were then used to monitor eye positions (Robinson, 1963). A computer controlled all the stimulus presentations, monitored the eye position and behavioral re- 


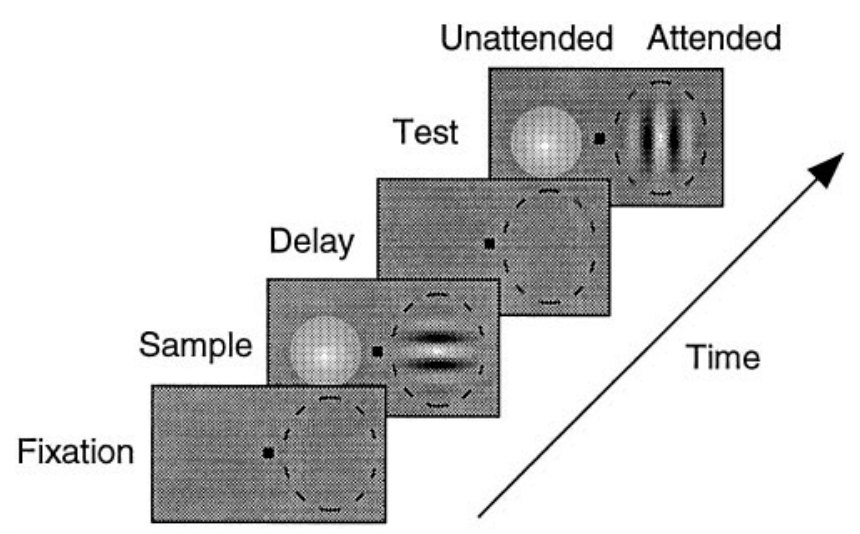

Figure 1. Schematic representation of the delayed match-to-sample task. Each frame represents the display at a different point in a trial, with the fixation spot in the center and the receptive field of the neuron indicated by a dashed oval. The fixation, sample, and delay periods were each 500 msec. The test period could last $1000 \mathrm{msec}$, but ended when the animal released the lever. The monkey was required to bring his gaze to the fixation spot and depress a lever to begin the trial. A Gabor and a colored Gaussian were presented in the sample period. The monkey attended to only one of these stimuli in each trial, based on previous instruction trials in which only one stimulus appeared. In the attended mode, the monkey was required to pay attention to the orientation of the stimulus in the receptive field. In the other mode, the monkey was required to pay attention to the color of the stimulus outside the receptive field. Both stimuli were removed during the delay period. In the test period, the animal had to report whether the test stimulus at the attended location matched the sample stimulus previously presented there. In the case illustrated, if the animal had been instructed to pay attention to the oriented stimuli, the animal would be required to keep the lever depressed to receive a juice reward because the orientations do not match. Conversely, if the animal had been instructed to pay attention to the colored stimuli, the animal would be required to release the lever within $500 \mathrm{msec}$ of the test stimulus onset to receive juice because the colors match.

sponses of the animal, and recorded the behavioral and neuronal data. Stimuli were presented on a color video monitor, positioned $70 \mathrm{~cm}$ from the animal.

The animals performed a delayed match-to-sample task (Fig. 1). The trial began when the animal looked at the fixation point and depressed a lever. Both animals were required to keep their gaze within $0.7^{\circ}$ of the center of the fixation point throughout the trial. After $500 \mathrm{msec}$, sample stimuli appeared at two different locations. Only one location was behaviorally relevant on a given trial. After a sample presentation of 500 $\mathrm{msec}$, the stimuli were removed for a $500 \mathrm{msec}$ delay period. Then two test stimuli appeared, at which point the animal had to indicate whether the test stimulus at the relevant location matched the sample stimulus previously presented at that location. If the test stimulus matched, the animal had to release a lever within $500 \mathrm{msec}$ of its onset to receive a reward. If the test stimulus did not match the previously presented sample, the animal had to keep the lever depressed for 750-1000 msec, after which time he received a reward.

The animal was trained to attend only to stimuli in one location on a trial. The relevant location was cued using instruction trials in which stimuli were only presented in one location. After the animal performed two instruction trials correctly the stimuli at the second location were reintroduced. The stimuli at one location were Gabors. The Gabors were constructed by multiplying a sinusoidal grating and a two-dimensional Gaussian. The contrast of these stimuli was modulated sinusoidally in time at $4 \mathrm{~Hz}$, although the mean luminance of the stimulus averaged over space and time was the same as the background. The animal had to report whether the sample and test Gabor orientations matched when instructed to attend to that location. The stimuli at the other location were isoluminant colored patches whose saturation varied with a twodimensional Gaussian profile. The animal had to report whether the sample and test colors matched when instructed to attend to that location. Matches and nonmatches at the two locations were uncorrelated so that the animal could get no advantage from attending to the wrong location. Animals were instructed to shift their attention from one location to the other after correctly completing 24 trials using one location. The first animal, A, had no problems remembering which location he was supposed to attend to, and he only received two instruction trials for each shift in location. The second animal, B, was more easily distracted and occasionally extra instruction trials were required within a block. A single instruction trial was given to him whenever he missed or ignored three trials in a row.

By presenting the same visual stimuli to the animal when he was performing the color matching task and when he was performing the orientation matching task, differences in neuronal responses occurring between the two types of trials could be attributed to differences in behavioral state between the tasks. The Gabors were always placed inside the receptive field of the neuron being recorded. The Gaussian patches were placed outside the receptive field of the neuron, diametrically opposed at the same eccentricity. Although the animal was attending to something in both task modes, we define an attention difference with respect to whether the neuronal signals we recorded were relevant or irrelevant to the current task. Thus, when the animal performed the orientation matching task, the neuron from which we recorded was responding to the relevant stimulus, so we refer to this mode as the "attended" mode. When the animal performed the color matching task, the neuron was still responding to the Gabor, but because this stimulus was now irrelevant to the animal's task, we refer to this mode as the "unattended" mode.

Attention effects in area V4 have been attributed to both spatial attention (Moran and Desimone, 1985; Motter, 1993; Connor et al., 1996, 1997) and to feature-directed attention (Maunsell et al., 1991; Motter, 1994a,b). The attentional modulation that we measured with this task design could have been caused by either of these forms of attention because the attended and unattended stimuli differed in both location and relevant dimension (orientation or color). We chose this design to increase the chances of encountering attentional modulations in area V4.

Neuronal recording and data collection. In both animals data were collected from V4, with additional recordings made in V1 for comparison. After the animals were trained, a recording chamber $(20 \mathrm{~mm}$ diameter) was implanted on intact skull overlying the operculum of V1. When recordings were completed, this chamber was removed, and a new chamber was positioned over V4. Animal A received a second V4 chamber, so that data were collected from three hemispheres in two animals. Recordings were usually made daily during a 3-5 week session. At the start of each session, a 5-8 mm craniotomy was made inside the chamber leaving the dura mater intact. Two or three craniotomies were made in each chamber. Each day a hydraulic microdrive was mounted on the recording chamber, which was then filled with sterile mineral oil and sealed. Transdural recordings were made using Pt/Ir recording electrodes of 1-2 M $\Omega$ at $1 \mathrm{kHz}$ (Wolbarsht et al., 1960). A small fraction of the data (30 of 262 cells) was recorded from the parts of V4 in the superior temporal sulcus using guide tube recordings with similar electrodes. Signals from the microelectrode were amplified, filtered, and monitored on an oscilloscope and audio monitor using conventional equipment. Recordings were made only from cortex within $3 \mathrm{~mm}$ of the surface using the transdural electrodes and up to $\sim 6 \mathrm{~mm}$ from the surface using guide tubes in one chamber.

The animal performed the match-to-sample task while we searched for responses. Units were isolated on the basis of waveform, with the requirement that the peak of the action potential be at least three times the background noise. When a unit was isolated, its receptive field was mapped with a bar moved by hand while the animal fixated a small spot of light. The receptive fields of the neurons were between 1 and $5^{\circ}$ eccentric. The Gabor stimuli were then adjusted in spatial frequency, color, and size to yield the best response using the match-to-sample task, as judged by listening to the audio monitor. The spatial frequencies used ranged from 1 to 5 cycles $/^{\circ}$. The size of the stimuli were taken as the SD of the Gabor and ranged from 0.6 to $2.2^{\circ}$. The spatial frequency and SD were varied independently; the range of the ratios of spatial frequency to $\mathrm{SD}$ was $0.8-8.3{\text { cycles} / \text { degree }^{2} \text { with a median of } 2.2 \text { cycles/degree }}^{2}$. Colors for the Gabors were selected from five options (black/white, blue/yellow, red/green, cyan/red, and magenta/green). Perhaps one in five neurons did not have any obvious orientation tuning or could not be driven well and was not examined further. For $\sim 15 \%$ of the neurons recorded, the animal would not work using the best stimulus we could find for that neuron. These stimuli were typically high spatial frequencies or small for their eccentricity. For those neurons, a suboptimal stimulus was used, provided that the neuron remained selective for orientation. 
Once stimulus parameters were set, those parameters were used for all data collected from the neuron.

Data analysis. We measured neuronal responses during the presentation of the sample stimulus. We collected at least eight repetitions of twelve orientations in each of the two task modes, in which task mode is defined by whether the animal attended to the stimulus inside (attended mode) or outside (unattended mode) the receptive field. Only correctly completed trials, excluding instruction trials, were counted and used in data analysis. Task mode alternated after obtaining two repetitions of each of the twelve orientations; so for most units four cycles of the attended and unattended task modes were collected. Undriven activity was estimated from the activity of the neuron during the period while the animal was fixating a central spot and before a stimulus was presented in the receptive field.

Orientation-tuning curves from cortical neurons are generally well represented by a Gaussian function (Henry et al., 1973; Geisler and Albrecht, 1997). We constructed orientation-tuning functions for each task mode by fitting the averaged responses for each orientation with a Gaussian using a nonlinear least squares optimization procedure that incorporated both the variance and the magnitude of the responses (Levenberg-Marquardt method, Press et al., 1989). The Gaussians had four free parameters: amplitude, SD, asymptote, and mean. We used the Gaussian SD as a measure of tuning width and the mean as a measure of the preferred orientation of the cell. The quality of fits was dependent on both the averaged responses and the variability of the responses to each orientation. The acceptability of each Gaussian fit was determined by performing an $F$ test that compared the goodness of fit from the Gaussian with the goodness of fit obtained from a line of any slope. Only the neurons meeting a minimal criterion for a good fit $(F$ test; $p<0.05)$ were used in comparisons across the two task modes; the median $r^{2}$ for these fits was 0.40 . To determine statistical significance of the changes in the fitted parameters across the two task modes for individual neurons, a set of parameters for each mode was produced by fitting a Gaussian tuning curve for each of the stimulus repetitions. A Mann-Whitney $U$ test was then performed on each parameter to determine which units had individually significant changes in amplitude, width, preferred orientation, and asymptote.

Additionally, a two-factor ANOVA was performed on each neuron to confirm the presence of both orientation tuning and attention modulations independent of fitting procedures. The ANOVA was performed by sorting the responses of each neuron by the orientation of the sample Gabor and the behavioral mode of the animal and then determining the amount of the total variance that could be attributed to the sample orientation and the behavioral (attentional) state. Neurons were considered to have individually statistically significant changes if the ANOVA resulted in a $p<0.05$.

Eye position analysis. Our task required that the monkey maintain fixation within $0.7^{\circ}$ of a central spot throughout the data collection period. Although identical visual stimuli were presented in the two behavioral tasks, differences in the responses might have resulted from small systematic differences in the animals' eye positions within the fixation window between the two tasks. This possibility is a concern because the two tasks differed in location of the attended stimuli. Several factors suggest that eye movements were not a problem. First, we measured the difference in fixation position for each task mode for each neuron recorded. The median fixation position difference across the two tasks was $<0.1^{\circ}$ in both animals. Second, the stimuli were selected to reduce the effects of small differences in eye position between the tasks. We chose Gabors and Gaussians because they have relatively little high spatial frequency content and this should help reduce the potential of microscopic eye movements to affect the neuronal response (Parker and Hawken, 1985; Shapley and Victor, 1986). Also, the stimuli were isoluminant with the background of the monitor over space and time, which should also reduce the chances of small stimulus offsets causing a systematic change in response.

Histology. When the experiments were completed, each animal was deeply anesthetized, and fiducial pins were inserted into V4 using the same equipment that had been used to position the microelectrodes. The animal was then anesthetized with an overdose of barbiturates and perfused with PBS followed by $4 \%$ paraformaldehyde.

Reconstruction of the recording regions based on the pins showed that all recordings were in V4 in the anterior part of the prelunate gyrus, dorsal to the end of the inferior occipital sulcus. Deeper recording made with guide tubes were located on the posterior bank of the superior temporal sulcus. No reconstruction was made of the $\mathrm{V} 1$ recording sites, which were from the opercular region well away from the vertical meridian representation that defines the V1/V2 border.

\section{RESULTS}

\section{Effects of attention on orientation-tuning curves}

We examined the effects of attention on the orientation tuning of 262 isolated neurons in area V4 in two monkeys. We selected orientation-tuned neurons and excluded an estimated $20 \%$ of the neurons we isolated because we could not drive them differentially with different orientations of a Gabor. The average rate of firing during the presentation of the sample stimulus was significantly modulated by orientation for $85 \%$ (223 of 262) of the recorded neurons and by attention for $55 \%$ (145 of 262) of the recorded neurons (two-factor ANOVA; $p<0.05$ ). Neurons with significant effects for both orientation and attention made up $47 \%$ (122 of 262) of the neurons, consistent with these properties occurring independently. Most neurons with significant effects of attention had increased firing rates in the attended condition $(86 \% ; 125$ of 145$)$. The orientation-tuning data were well fit by a Gaussian in both behavioral conditions for $75 \%$ of the neurons (197 of 262; $F$ test; $p<0.05$ ). We will consider these cells here and take up the remaining cells later.

Data from a neuron showing a clear effect of attention are shown in Figure 2. The histograms (Fig. 2A) plot the responses around the time the sample stimulus was presented (shaded). The top row shows the responses obtained during the attended trials; the bottom row contains the corresponding data from the unattended trials. Responses to each of the orientations were stronger when the animal was attending to the stimulus, with greater response increments for stronger responses.

Figure $2 B$ plots the average firing rate of this neuron as a function of orientation. Gaussians were fitted separately to the attended and unattended responses by varying four parameters: amplitude, SD, asymptote, and preferred orientation. The amplitude of the fitted function for the attended condition was $43 \%$ greater (57 spikes/sec vs 40 spikes/sec; $p<0.001$, Mann-Whitney $U$ test, see Materials and Methods), but there were no significant changes in the fitted width, asymptote, or preferred orientation $(p>0.05)$. This result was typical of the cells we sampled.

The distributions of change in the tuning parameters for V4 neurons are displayed in Figure 3. The change in the amplitude of the orientation-tuning curves associated with attention is shown in Figure $3 A$. The median ratio in our population is 1.26 , indicating that attention systematically strengthened responses to the attended stimuli. This increase was significant (Wilcoxon signed rank test; $p<0.001)$. Attention produced individually statistically significant changes in amplitude in $20 \%$ of the cells (39 of 197; Mann-Whitney $U$ test; $p<0.05)$. These are marked in black in the histogram. All but two of the cells with significant changes had increases in response strength by attention. These results are consistent with previous studies demonstrating that attention increases neuronal firing rate.

One question we sought to answer was whether attention systematically makes tuning curves narrower, thereby providing a more precise representation of orientation. Figure $3 B$ shows the change in orientation-tuning width (SD of the fitted Gaussian) for the population. If attention narrowed tuning curves, the distribution would be shifted to the left. However, our population is evenly distributed around a ratio of 1.0, which corresponds to no change in width (Wilcoxon signed rank test; $p>0.5$ ). Only $9 \%$ of the neurons showed individually significant changes in width 

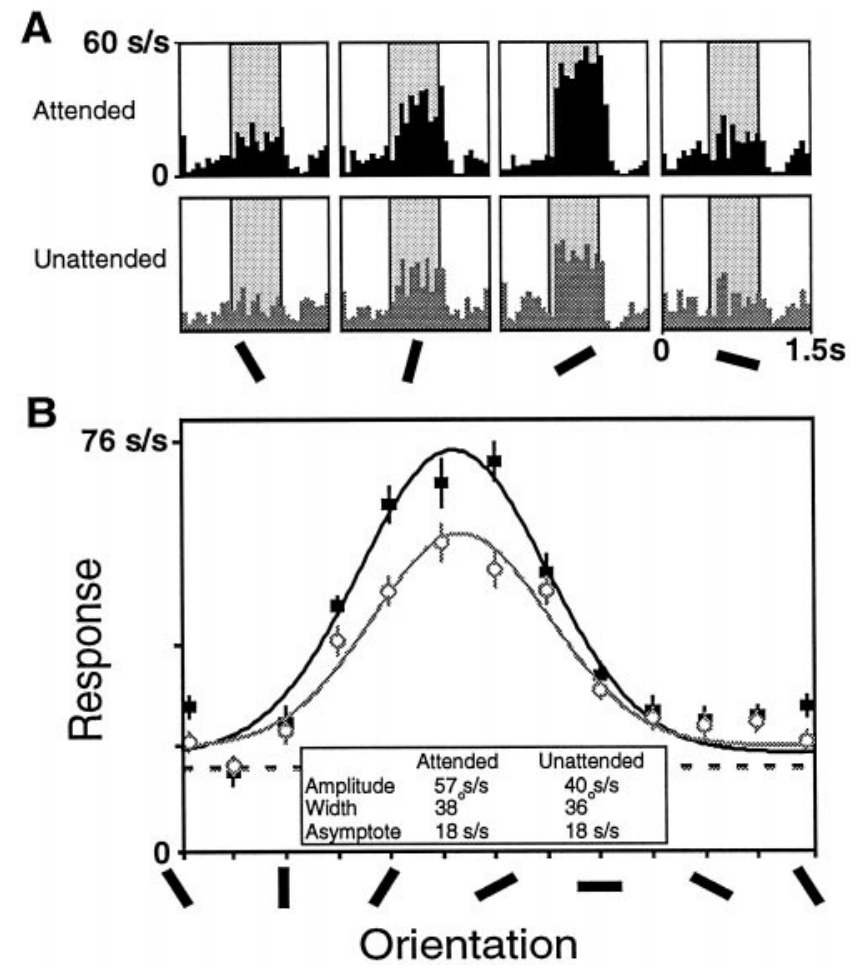

Figure 2. Data from one V4 cell showing enhanced responses in the attended mode (black) relative to the unattended mode (gray). A, Histograms showing the responses elicited by sample stimuli of four different orientations. The histograms in the top row were taken from trials when the animal was attending to the receptive field stimulus, and the histograms in the bottom row were taken from trials when the animal was attending to the stimulus outside the receptive field. The average response during the sample period (shaded) was used to construct tuning curves. $B$, Tuning curves were constructed for this neuron for each task mode by fitting the responses for each condition to a Gaussian. This cell had a significant increase in amplitude in the attended mode (solid symbols) relative to the unattended mode (open symbols), but no significant changes in the preferred orientation, width, or asymptote. The undriven activity of the cell during the attended trials is shown in the black dashed line, and the undriven activity of the cell during the unattended trials is represented by the gray dashed line.

(Mann-Whitney $U$ test; $p<0.05$ ), and those cells included about as many width increases as width decreases.

The asymptote of the orientation-tuning curve reflects the responses of the neurons to the least preferred orientations. Figure $3 C$ shows the change in the orientation-tuning asymptote with attention. Although the median ratio is 1.0, the population is skewed to the right and shows a statistically significant increase in asymptote with attention (Wilcoxon signed rank test; $p<0.005$ ). Individually significant changes in asymptote were found in $13 \%$ (25 of 197; Mann-Whitney $U$ test; $p<0.05$ ) of the cells, and most of those cells (21 of 25 ) show increases in asymptote.

The final parameter of the tuning curve is the preferred orientation of the cell. Figure $3 D$ plots the difference between the preferred orientations for the two conditions. The axes of this plot are different than those in the other panels. There was no reason to expect a change in the preferred orientations by attention, and the median difference in fitted orientation for the two modes was well under $1^{\circ}$ and not significantly different than no change (Wilcoxon signed rank test; $p>0.29$ ). Only $8 \%$ of the cells showed individually significant changes in preferred orientations (Mann-Whitney $U$ test; $p<0.05$ ).
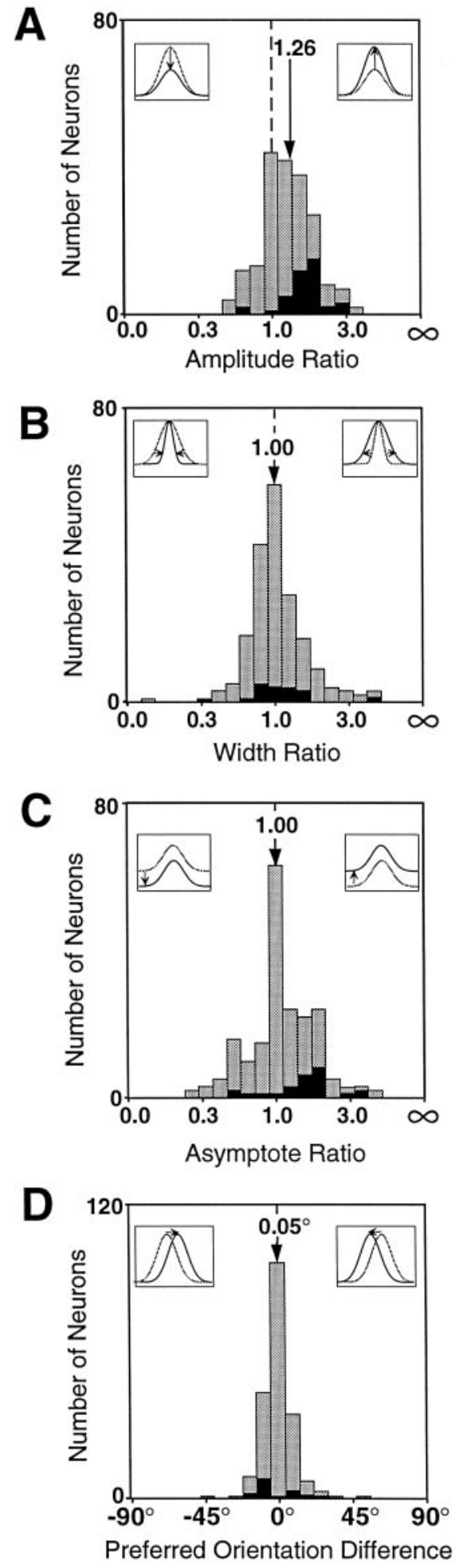

Figure 3. The changes in the parameters of orientation tuning by attention are characterized using index values for the amplitude $(A)$, width $(B)$, asymptote $(C)$, and the difference in preferred orientation $(D)$. The index value is the difference in the fitted Gaussian parameters, (attended - unattended) divided by their sum (attended + unattended). The index value was selected for binning because it is bounded for both positive and negative changes, but the axis is labeled in the corresponding ratios. All of the plots display the changes in the fitted parameters for the 197 V4 neurons whose orientation-tuning curves were well fit by a Gaussian. Cells showing individually significant changes in a given parameter are drawn in black. 


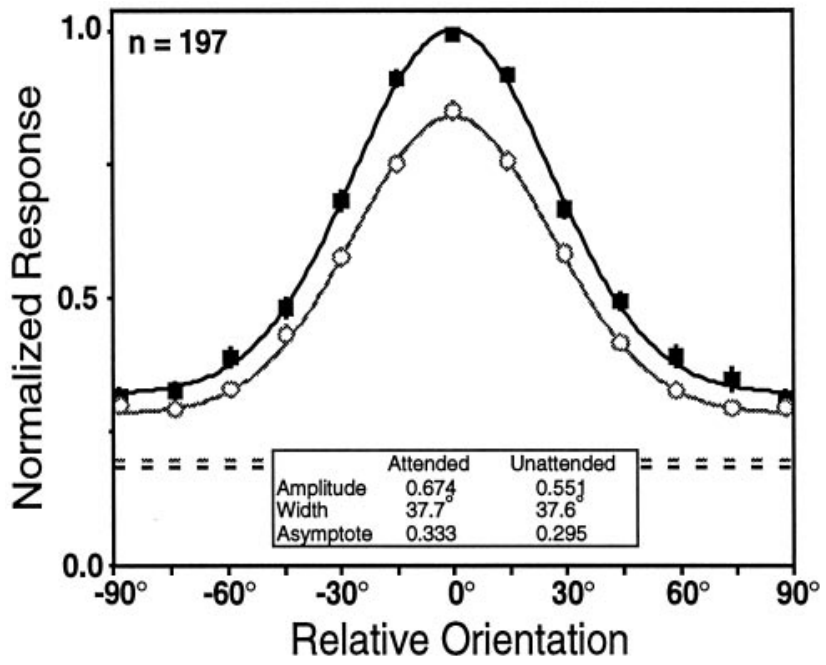

Figure 4. The population-tuning curves for the V4 neurons that were tuned in both the attended (solid squares) and unattended (open circles) task modes. The preferred orientation for each cell was estimated for the purpose of aligning the population-tuning curves by smoothing the data sets of each neuron, fitting Gaussians to the smoothed data for each task mode, and then averaging the fitted attended and unattended preferred orientations. Gaussians were then fit to the averaged data for each behavioral mode. The dashed lines represent the average undriven activity, measured as the firing rate during the fixation period for all trials for each task mode, with the darker line corresponding to the undriven activity during the attended mode and the lighter line corresponding to the undriven activity during the unattended mode.

\begin{tabular}{lcc}
\hline Table 1. Attention effects on orientation tuning in V4 & \\
V4 & Unattended & Attended \\
\hline Amplitude (spikes/sec) & 12.0 & 14.8 \\
Width $\left({ }^{\circ}\right)$ & 39.2 & 37.3 \\
Asymptote (spikes/sec) & 4.7 & 4.7
\end{tabular}

The median Gaussian values for the attended and unattended modes for all neurons with acceptable tuning in both modes from area V4 $(n=197)$.

Another way to visualize the overall changes in orientation tuning associated with attention is to construct population-tuning curves for the two task modes. The responses in Figure 4 were determined by aligning the responses of each cell on its preferred orientation, normalizing responses to the response in the attended mode at the preferred orientation, and then averaging the responses to different stimuli and task modes across cells. These curves provide a picture of the average change in orientation tuning produced by attention among orientation-tuned cells in area V4. They show a $22 \%$ increase in amplitude with attention and a $13 \%$ increase in asymptote with attention, with no appreciable change in the width or preferred orientation.

In summary, attention appears to scale the entire orientationtuning function. Table 1 contains the median fitted values for amplitude, width, and asymptote for the 197 V4 cells showing orientation tuning in both task modes. The possibility that differences in fixation or eye movements of the animals across the behavioral states might contribute to this effect is addressed in a later section.

\section{Cells that lacked orientation tuning}

The preceding analysis treated the $75 \%$ of the population that had statistically significant Gaussian orientation tuning for both

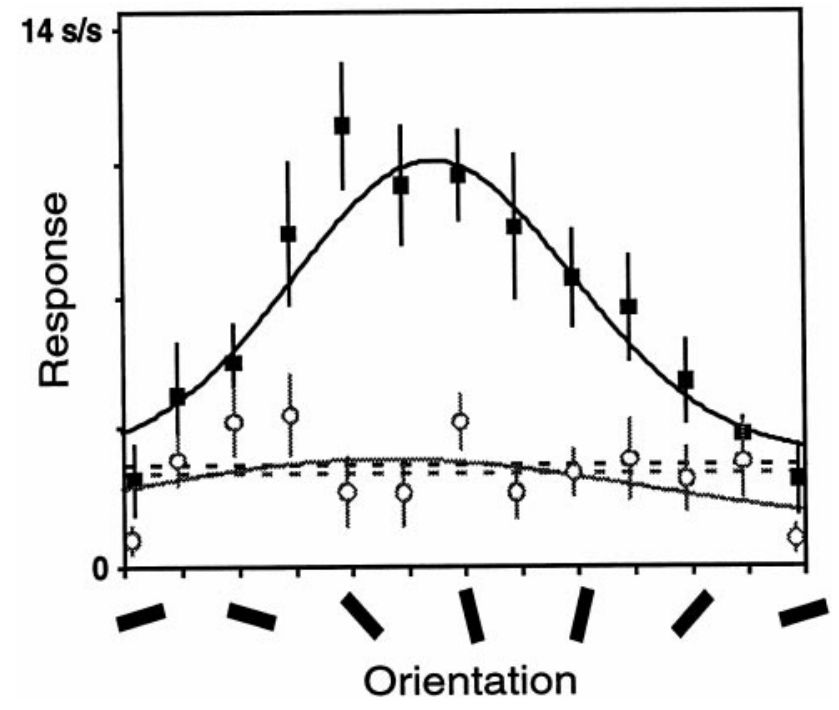

Figure 5. Tuning curves for a single V4 unit that had acceptable orientation tuning in the attended mode (black) but not in the unattended mode (gray). Same format as in Figure $2 B$.

the attended and unattended task modes. Another subset of the V4 neurons only had acceptable Gaussian tuning fits in the attended mode but not in the unattended mode. These cells comprised 16\% (42 of 262) of the population.

Responses from one such neuron are shown in Figure 5. The fit of the tuning curve is acceptable in the attended mode ( $F$ value of $10.4 ; p<0.001)$ but not in the unattended mode ( $F$ value of 1.4; $p>0.50)$. Parameters from an unacceptable fit usually do not provide meaningful information. For this neuron, the fitted widths are $53^{\circ}$ in the attended condition and $94^{\circ}$ in the unattended condition. Taken at face value, these values suggest a dramatic narrowing of orientation tuning by attention, and the average width ratio for this subset of cells corresponds to a $51 \%$ narrowing of orientation tuning by attention. This apparent narrowing is misleading because the data for the unattended condition are comparably well fit by a straight line, and therefore could be described as tuning curves that have approached zero amplitude with no change in width.

One way to assess whether attention leads to a genuine narrowing of the low amplitude tuning curves is to improve their signal-to-noise ratio by averaging their responses before fitting functions to the data. Figure 6 shows the result of creating population-tuning curves by averaging the 65 neurons that failed to meet the criterion for a Gaussian fit in either or both task modes, using the same methods as in Figure 4. These neurons include 42 cells with acceptable tuning in the attended mode only, 17 cells that were not acceptably tuned in either mode, and 6 cells that had acceptable tuning only in the unattended mode. The combined responses show a large attention effect, with the amplitude more than doubling. However, the width of the two curves is not appreciably different.

Normalized population-tuning curves constructed from all 262 neurons recorded from area $\mathrm{V} 4$, tuned and untuned, are shown in Figure $7 A$. This figure differs from Figure 4 in that it includes data from every V4 neuron examined rather than only those showing significant tuning in both conditions. The resulting curves show the overall changes in orientation tuning that attention produces across all cells in V4. Attention leads to a $31 \%$ increase in the 


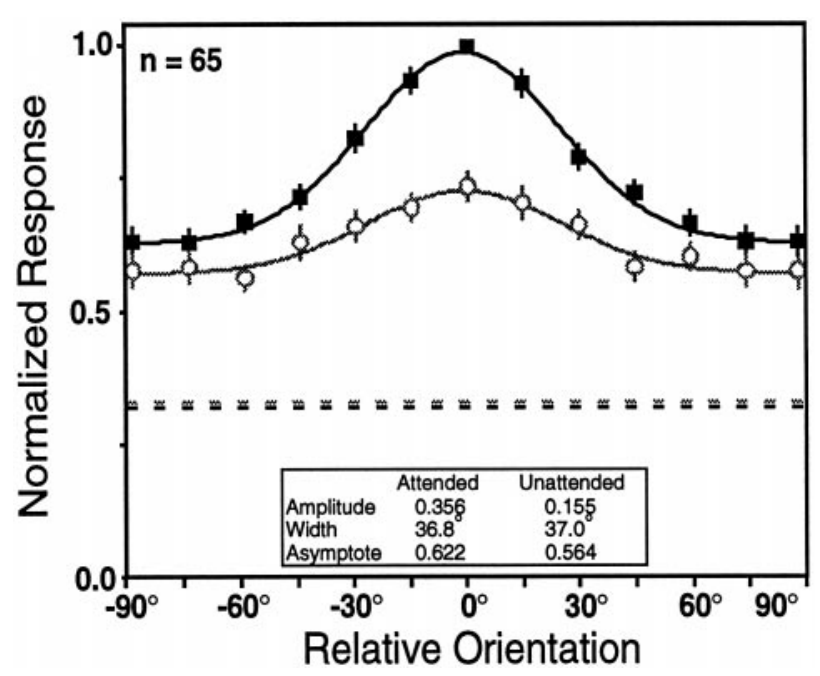

Figure 6. Population-tuning curves for the V4 neurons that were not well fit by a Gaussian tuning curve in one or both task modes. Same format as in Figure 4. Both the attended (black) and unattended (gray) populationtuning curves reach our criterion for orientation tuning. Although there is a large difference in amplitude, there is little difference in the width of the curves.

amplitude, a $13 \%$ increase in the asymptote, and no appreciable change in width in the population tuning curve.

\section{Multiplicative scaling}

A multiplicative scaling of orientation-tuning curves by attention, without a change in tuning width, would be reminiscent of the response changes that have been described in V1 and elsewhere when a stimulus parameter such as luminance or contrast is varied (see Discussion). For example, increasing the contrast of a sinusoidal grating typically increases the response of a neuron to all orientations proportionately, so that a tuning curve constructed from responses to high-contrast stimuli is a multiplicatively scaled version of one made using lower contrast stimuli (Dean, 1981; Sclar and Freeman, 1982; Skottun et al., 1987).

An amplitude change with no width change is consistent with multiplicative scaling, but can occur when scaling is not multiplicative. Whether scaling is multiplicative can be tested by examining whether the neuronal responses at each orientation change proportionately. Figure $7 B$ replots the data from Figure $7 A$, such that the normalized population response in the attended mode is plotted against the normalized population response in the unattended mode. If attention causes a proportional change in the evoked response, these data should be well fit by a straight line. The slope of a linear regression of the attended responses on the unattended responses is 1.32 , reflecting a $32 \%$ enhancement of the neural responses by attention. The correlation coefficient for these points is 1.00 , strongly supporting the notion that attention produces proportional changes in the stimulus response. Additionally, the fitted line intersects the undriven activity of the neurons (dashed lines), which is also expected if attention produced a multiplicative scaling of the evoked responses without scaling the undriven activity of the neurons.

\section{Time course}

Several earlier experiments have examined effects of attention on time course in area V4 using different experimental paradigms. Motter (1994a) reported a 150-200 msec delay after stimulus onset for the emergence of an attention effect, using an experi- ment in which attention was directed to a particular feature before the onset of a stimulus array. Other studies separated the effects of attention from stimulus responses more completely by providing an attention cue that selects some stimuli from an array after the array is present. In these studies, attentional effects appeared between 50 and $300 \mathrm{msec}$ after the attention cue was provided (Motter, 1994b; Preddie et al., 1995; Bricolo et al., 1997; Makous et al., 1997). In our experiments, the animal was cued to attend to a spatial location well before stimulus onset, so that an attention effect might be present during the earliest portion of the response.

In Figure $8 A$, population histograms of the responses to the preferred orientation of each cell in the attended and unattended modes are shown. These histograms were constructed by averaging the responses to the preferred orientation for every neuron for each task mode. The periodicities in the response correspond to half-cycles of the counterphasing of the Gabor stimulus $(4 \mathrm{~Hz})$ that were visible in the responses of some cells. Figure $8 B$ shows the ratio of the responses in Figure $8 A$ as a function of time. This ratio is a measure of attentional modulation and slowly rises from the stimulus onset to reach a value of $\sim 1.5$ of the unattended response. The $31 \%$ increase reported above is effectively the time average of this curve. This time-plot of the attention effect shows that although the relevant location was known before stimulus onset, the effects began after the stimulus was presented and increased during the $500 \mathrm{msec}$ stimulus presentation period.

The results presented in Figure $7 B$ show that attention systematically and multiplicatively scales responses across orientations regardless of the magnitude of the response. Another issue is whether there is a multiplicative scaling for responses to different orientations across time. This can be examined by comparing population histograms of the attended and unattended responses as a function of orientation. The time course of the ratio of the attended response to the unattended response for three other orientation differences relative to the preferred orientation are also shown in Figure $8 C$. The attention input has a similar time course and reaches approximately the same level of enhancement for each stimulus condition.

Because our task used a block mode in which the animal attended to one spatial location for at least 26 trials before attention was directed to the other location, the activity of the cells could have been modulated by attention during periods when no stimulus was present. Although the population histograms in Figure 8 suggest that attentional modulation did not begin until the stimulus appeared, this question can also be examined in the single cell data. In Figure 9, the undriven activity of each neuron in the attended mode is plotted against its undriven activity in the unattended mode. Although $24 \%$ of the neurons (63 of 262) showed individually significant changes in undriven activity ( $t$ test; $p<0.05)$, those changes included a similar number of increases and decreases (filled circles). The population also shows no statistically significant difference between the undriven activity in the attended mode and the unattended mode in our task (Wilcoxon signed rank test; $p>0.5$; median 3.6 spikes/sec attended mode, 3.6 spikes/sec unattended mode), and the best fit line has a slope close to one (slope 0.92; $r=0.98)$. Thus, the attention effect was evident in area V4 only in the presence of a visual stimulus. Luck et al. (1997) reported that spatial attention increased the undriven activity of V4 neurons by an average of three spikes per second. Those investigators used a different behavioral task and found an overall higher level of undriven activity (15 spikes/sec). 
A

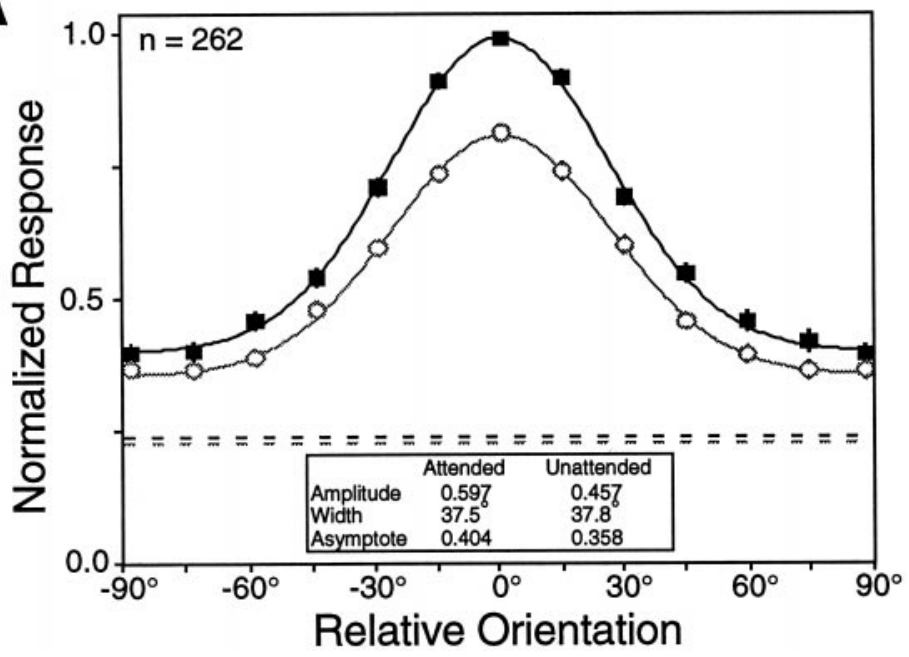

B

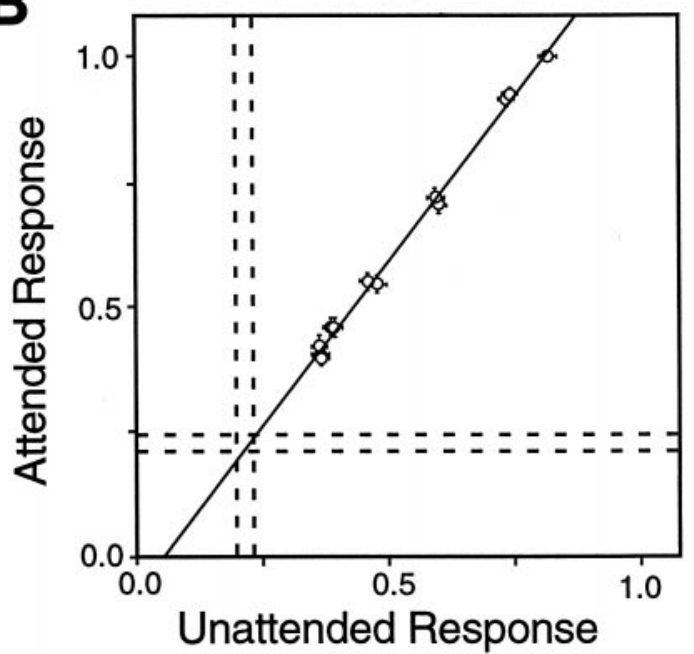

Figure 7. A, The normalized population-tuning curves for all V4 neurons. Same format as in Figure 4. Although there is a large difference in amplitude, there is little difference in the widths of the curves. $B$, The attended response is plotted against the unattended response for each of the 12 orientations. The line shown is the linear regression of the attended responses on the unattended responses. It has a slope of 1.32 , corresponding to a $32 \%$ increase in response with attention. The pairs of dashed lines mark \pm 1 SEM undriven activity. The excellent fit $\left(r^{2}=1.00\right)$ and the intersection with the undriven activity are consistent with a multiplicative scaling of the evoked responses.

\section{Behavioral performance}

We found differences in performance of the animals across our two task modes, with both animals performing worse on orientation (attended mode) than on color (unattended mode). Collapsed over all data-collection trials from each animal (but excluding those trials in which the animal broke fixation or ignored outright), animal A's performance was $83 \%$ on the orientationmatching task and $92 \%$ on the color-matching task, and animal B's performance was $82 \%$ on the orientation-matching task and $88 \%$ on the color-matching task. The difficulty of each task depends on how different the nonmatching stimulus is from the matching stimulus. For the orientation task, this is primarily determined by the rotation between the sample and test orientations. This rotation was $45^{\circ}$ for animal A and $90^{\circ}$ for animal B. We fixed the orientation difference and then tried to adjust the animal's performance in the color task to a similar level. The color stimuli were selected to be isoluminant with the gray background screen. Five sample colors were available: pink, red, yellow, green, and cyan. The test colors were created by slightly changing the sample hue. We were unable to adjust the color difference finely enough to balance the performance on the color task with that on the orientation task.

Because differences in task difficulty alone can produce attention effects (Spitzer et al., 1988; Spitzer and Richmond, 1991), we were interested in whether the attention effect that we measured could be caused by the difference in difficulty across task modes. One approach to this question is to determine whether cells showing the largest attentional modulations were those recorded while the animal had large differences in performance between the task modes. Because the Gabor stimulus was repositioned and reconfigured for each cell, behavioral performance varied from cell to cell. An attentional modulation index (attended amplitude - unattended amplitude)/(attended amplitude + unattended amplitude) and a performance index (attended performance - unattended performance)/(attended performance + unattended performance) were calculated for each cell. Although the correlation was low, it was significant $(r=0.13 ; p=0.04)$, but
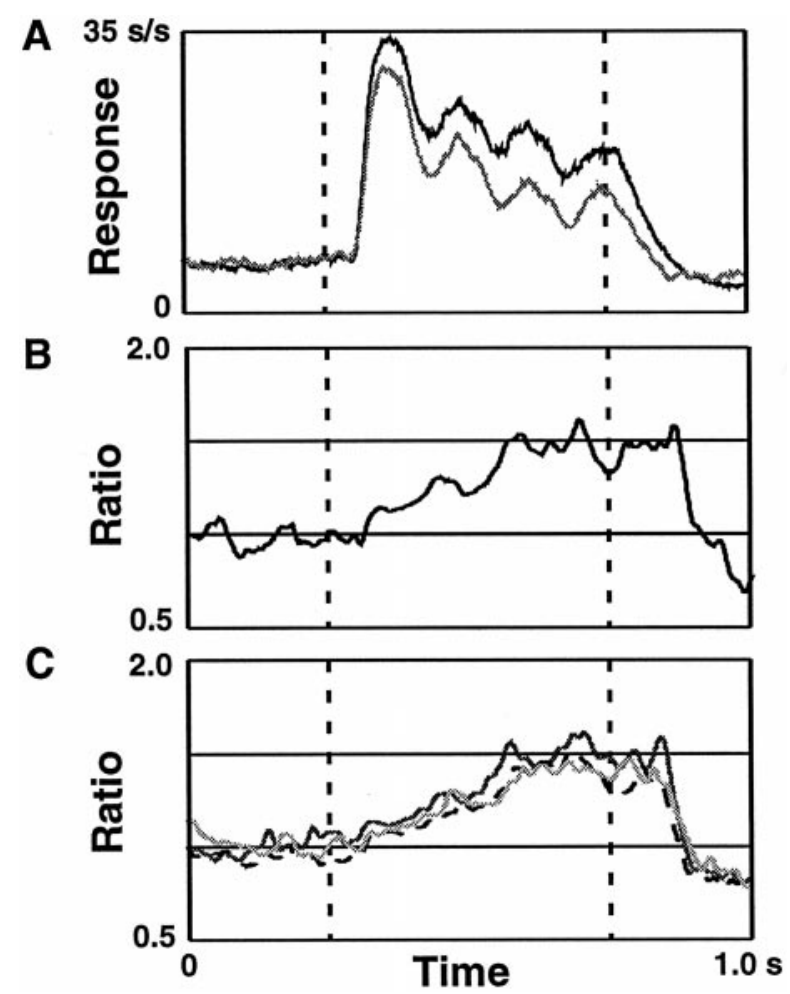

Figure 8. The time course of the attentional effect. A, Population histograms of the activity in response to the preferred orientation are shown for the attended (black) and unattended (gray) modes. The dashed lines indicate stimulus start and stop. $B$, The ratio of the attended activity relative to the unattended activity from the population histograms in $A$ is shown. $C$, The ratios for three other stimulus orientations. The darkest line corresponds to a stimulus orientations $15^{\circ}$ away from the preferred orientation of each cell, the next darkest line is a stimulus orientation $45^{\circ}$ from the preferred orientation, and the lightest line corresponds to a stimulus orientation $75^{\circ}$ from the preferred orientation. Although these stimuli resulted in responses of different magnitudes, the attentional modulation has the same time course and same proportional enhancement. 


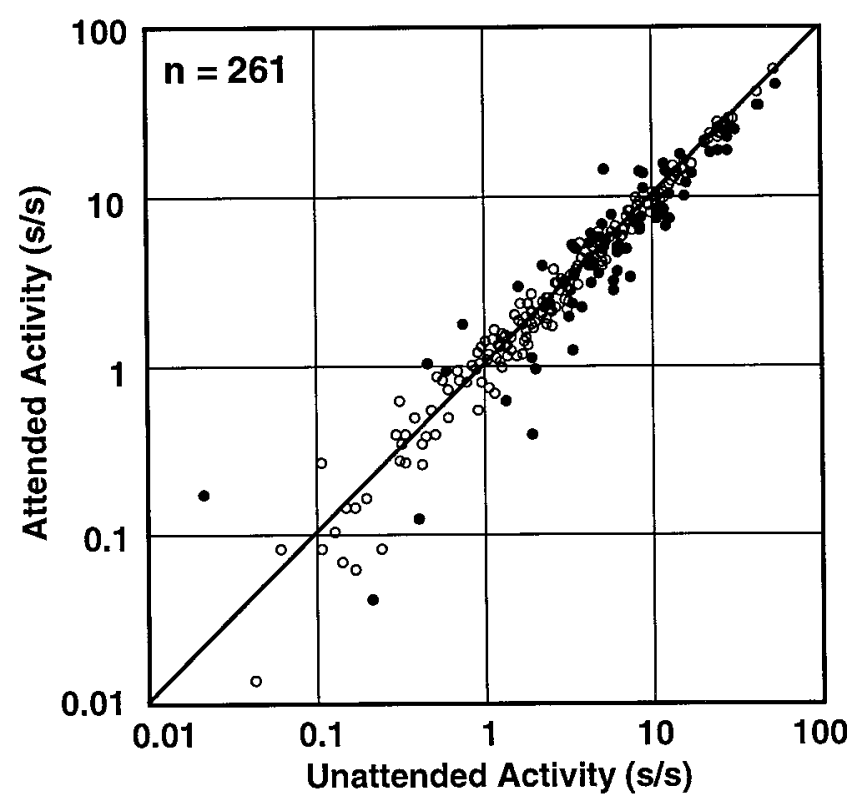

Figure 9. Undriven activity. The undriven activity for each neuron, measured as the average firing rate during the fixation period for the attended mode is plotted against the undriven activity generated in the unattended mode of the task. The solid circles are those cells with individually significant changes in activity $(t$ test; $p<0.05)$ across conditions. One V4 cell was excluded because it had zero undriven activity in one condition.

it was opposite to the direction expected if the attention effect depended on difficulty. This suggests that the attentional modulation described here stemmed mainly from selective attention for either spatial location, stimulus feature (color or orientation), or both.

\section{Effects in area V1}

We obtained orientation-tuning data from $135 \mathrm{~V} 1$ neurons. These data provide information about whether attentional modulations exist in the earliest level of cortical processing. Another reason for acquiring the $\mathrm{V} 1$ data were to provide an estimate of the magnitude of task effects that might be attributable to small differences in eye position. In principle, eye movements within a $1^{\circ}$ fixation window can change neuronal responses. Because the small V1 receptive fields can be extremely sensitive to stimulus offsets (De Valois et al., 1982), V1 neurons should be more susceptible to artifacts from small eye movements than neurons in areas like V4. Data from V1 can therefore serve as an upper estimate of possible eye movement contributions to the V4 data.

The V1 data were collected before recording the V4 data from each animal. This is relevant because both animals' fixation improved as recording progressed. The first animal had an average eye position difference across the two tasks of $0.09^{\circ}$ during the V1 data collection period but only a $0.04^{\circ}$ eye position difference during the V4 data collection period. For our stimuli, a $0.09^{\circ}$ offset corresponds to 4.1 pixels on the stimulus monitor; the fixation target was $8 \times 8$ pixels. The eye position offset across the tasks usually brought the animal's fovea closer to the target it was attending. The second animal had an average eye position difference of $0.10^{\circ}$ during the $\mathrm{V} 1$ data collection period and a $0.05^{\circ}$ eye position difference during the V4 data collection period. This animal's eye position offset across the tasks was not as dependent on the location of the attended stimulus, but appeared to be a stereotyped systematic fixation offset at two different locations for

\begin{tabular}{lll}
\hline \multicolumn{2}{l}{ Table 2. Attention effects on orientation tuning in V1 } \\
V1 & Unattended & Attended \\
\hline Amplitude (spikes/sec) & 21.0 & 22.1 \\
Width $\left(^{\circ}\right.$ ) & 35.2 & 37.6 \\
Asymptote (spikes/sec) & 4.1 & 4.4
\end{tabular}

The median Gaussian values for the attended and unattended modes for all neurons with acceptable tuning in both modes from area V1 $(n=125)$.

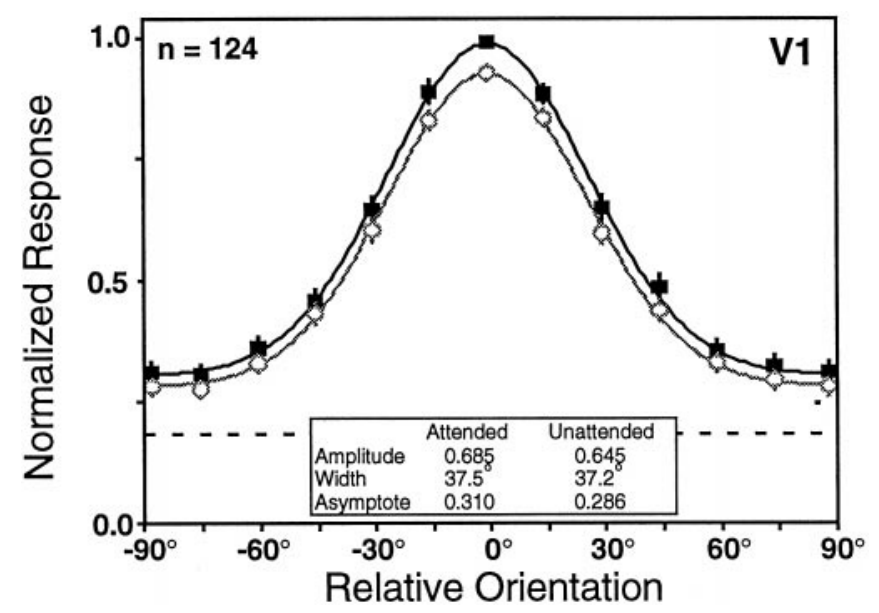

Figure 10. The population-tuning curves for V1 neurons for the attended (black solid squares) and unattended (gray open circles) task modes. Only 124 of the total $135 \mathrm{~V} 1$ neurons recorded could be used to construct these population-tuning curves because we used a $10^{\circ}$ sample spacing for 11 finely tuned V1 neurons, rather than the $15^{\circ}$ sample spacing used for all $\mathrm{V} 4$ and most of the V1 neurons. The dashed line indicates the relative magnitude of the undriven activity, which did not differ across the conditions. The overall increase in response amplitude in V1 was $\sim 6 \%$.

the two task modes. Thus, the V1 data are probably more likely to contain eye position artifacts that the V4 data, not only because $\mathrm{V} 1$ cells are more prone to artifacts but also because the animals offset their fixation more when the V1 data were collected.

The V1 data set shows small differences in the responses obtained during the two tasks. Attended and unattended orientation-tuning curves were recorded from $135 \mathrm{~V} 1$ neurons. As in V4, we selected for orientation-tuned cells and did not collect data from $\sim 20 \%$ of the cells we isolated. The average rate of firing during the presentation of the sample stimulus was significantly modulated by orientation for $99 \%$ (134 of 135) of the neurons and by attention for 31\% (42 of 135) of the neurons (two-factor ANOVA; $p<0.05$ ). Of the units showing significant task modulation, $83 \%$ (35 of 42) exhibited increased responses in the attended condition. The orientation-tuning data were well fit by a Gaussian in both behavioral conditions $(F$ test; $p<0.05)$ for 125 neurons. For these cells, we found that attention led to an $8 \%$ increase in tuning curve amplitude (Wilcoxon signed rank test; $p<0.001$ ), a $1 \%$ increase in width (Wilcoxon signed rank test; $p>0.15$ ), no change in asymptote (Wilcoxon signed rank test; $p>0.3$ ), and no change in preferred orientation (Wilcoxon signed rank test; $p>0.5$ ). A summary of the single cell data are provided in Table 2. The V1 population tuning curves in Figure 10 show a $6 \%$ increase in amplitude, an $8 \%$ increase in asymptote, and a $1 \%$ increase in width with attention. Other studies have reported attentional effects of comparable magnitude in area V1 (Motter, 1993; Press and Van Essen, 1997).

We cannot be sure whether our V1 effects are caused by eye 
position differences or whether they represent an attention effect. However, we can take the size of these V1 effects to be an upper limit on the size of effects that might be attributable to eye position artifacts for this particular data set. Because the measured median task effect in area V4 was approximately threefold greater than the task effect in area V1, we believe that the task effect in area V4 is caused by an extraretinal signal.

The systematic increase in responses in the attended mode hints that the V1 change arises from attention, because eye movements should not generally have a systematic effect. It would be unsafe to draw that conclusion, however. Although the receptive field was mapped while the animal was only fixating, many V4 neurons do not respond well during passive fixation (Maunsell et al., 1991). The Gabor was moved if it did not appear to be driving the cell well while the animal performed the task. Because instruction trials for the unattended mode do not present a stimulus in the receptive field, adjustments to the position of the Gabor stimuli were usually performed while the animal attended to the Gabor. Therefore, the stimulus may have been more optimally positioned for the fixation position occurring in the attended mode, leading to increased responses for the attended mode relative to the unattended mode.

\section{DISCUSSION}

\section{Orientation selectivity}

A primary goal of these experiments was to determine whether attention altered the orientation selectivity of neurons in macaque area V4. Our task revealed attentional modulation of the amplitude and the asymptote of orientation-tuning curves in area V4, but there was little evidence for change in the width of tuning. The principal action of attention appeared to be a multiplicative scaling of responses.

Previous studies of V4 suggested that attention narrows orientation-tuning and color-tuning curves (Haenny and Schiller, 1988; Spitzer et al., 1988). Although there are several sources that may contribute to this discrepancy, the main factor is likely to be the definition of width. Both earlier studies measured tuning curve width at some fraction of the peak of the curve, without compensating for the asymptote of the fitted curves, which will be nonzero if there is undriven activity or a response to the least preferred orientation. In the presence of a nonzero asymptote, this measure will yield narrower widths when responses above undriven activity increase proportionately. This behavior is illustrated in Figure $11 A$. The tuning curves in the top panels are multiplicatively scaled versions of the tuning curves in the bottom panel. The peak of each curve is measured as the height above either the undriven activity or a spike rate of zero (either represented by the $x$-axis). The half-width measured at half-height is larger in the bottom panel than in the top panel. This change in measured width will occur whenever there is a response to the least preferred orientation (tuning curve asymptote is above undriven activity). The measure we have used is the SD of a fitted Gaussian, that is, the curve width at a fraction between the maximal height and the base of the curve. This measure preserves width when responses increase proportionately. In Figure $11 B$, the height is measured as the amplitude of the Gaussians and is the difference between the peak response and the asymptote responses (dotted line). The half-width, measured at half-height, is the same in the top and bottom panels.
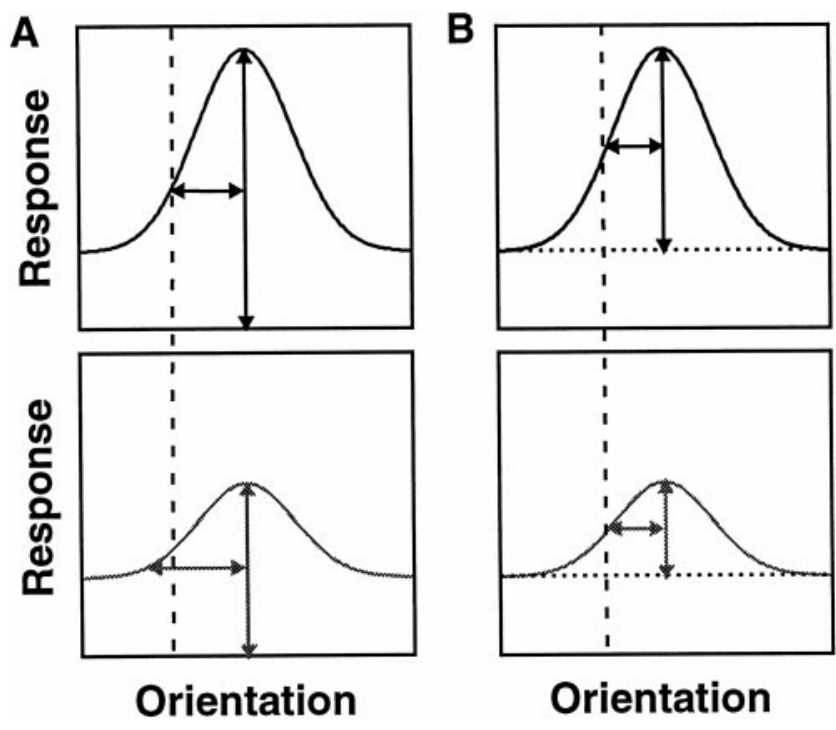

Figure 11. Effects of changes in response strength on different measures of width. The top panels are multiplicatively scaled versions of the tuning function in the lower panels. $A$, Height measured relative to undriven activity or zero activity. The vertical double-headed arrow is the assigned height of each curve, at which the $x$-axis represents either undriven activity or no activity. The horizontal double-headed arrow is the halfwidth at half-height. The width differs for the two curves (vertical dashed line). $B$, Height measured relative to the asymptote of the tuning function. The vertical double-headed arrow is the assigned height of each curve, at which the dotted line represents the asymptote of the tuning function. The horizontal double-headed arrow is the half-width at half-height. The width is the same for the two curves (vertical dashed line).

\section{Multiplicative scaling}

A multiplicative scaling of neuronal responses by attention is significant because this effect is also seen when stimulus parameters are manipulated. For example, changing the stimulus contrast can cause large changes in neuronal responses, but typically does not change the preferred orientation or sharpness of tuning. Instead, the orientation-tuning curve is scaled as contrast changes. Pairwise studies of many stimulus dimensions have shown this type of behavior. These include contrast and orientation or direction of motion (Dean, 1981; Sclar and Freeman, 1982; Skottun et al., 1987); contrast and spatial frequency (Dean, 1981; Holub and Morton-Gibson, 1981; Albrecht and Hamilton, 1982; Skottun et al., 1987; Geisler and Albrecht, 1997); contrast and spatial position (Geisler and Albrecht, 1997); orientation and direction of motion (Geisler and Albrecht, 1997); and spatial frequency and temporal frequency (Ikeda and Wright, 1972; Tolhurst and Movshon, 1975; Holub and Morton-Gibson, 1981; Bisti et al., 1985; Foster et al., 1985; Galli et al., 1988; Hamilton et al., 1989; Friend and Baker, 1993; McLean and Palmer, 1994a,b). One exception is direction selectivity, in which the responses to space and time that generate direction selectivity are not separable (Adelson and Bergen, 1985). Other neurons have sensitivities to spatial and temporal frequencies that interact, as in the case of preferring a particular speed (Ikeda and Wright, 1972; Foster et al., 1985; Friend and Baker, 1993). But even for those neurons, the interactions are often separable within individual quadrants of the frequency domain (Hamilton et al., 1989; McLean and Palmer, 1994a,b). Despite these exceptions, multiplicative scaling seems to be the most common type of interaction among stimulus attributes.

The current results suggest that the interactions between sen- 
sory signals and attentional modulations may also be multiplicative. Multiplicative scaling only affects responses to sensory stimuli; the undriven activity of the neuron was not changed by attention. Other types of extraretinal inputs to visual cortex also appear to cause a multiplicative scaling of sensory responses: signals related to the angle of gaze appear to scale receptive field profiles of neurons in posterior parietal cortex (Andersen et al., 1985); behavioral differences between reward contingent and nonreward contingent stimuli scale orientation-tuning curves in inferotemporal cortex (Vogels, 1994); and receptive field profiles of V4 neurons appear to scale multiplicatively when attention is directed to different points around the receptive field (Connor et al., 1996, 1997). Thus, multiplicative scaling might be a normal mode of interaction between all inputs to cortex. Although mechanisms that would produce multiplicative scaling have been described (Albrecht and Geisler, 1991; Carandini and Heeger, 1994), it is not known why such separability of response sensitivities may be important for cortical processing. Nevertheless, the phenomenological similarity between the effects of attention and the effects of stimulus manipulations raises the possibility that attention involves neural mechanisms that are similar to those used in processing ascending signals from the retinas, and that cortical neurons treat retinal and attentional inputs equivalently.

There is an important counterexample to multiplicative scaling by attention. The effects of attention on the spatial tuning of V4 neurons (i.e., the receptive field profile) has been examined in two studies. Moran and Desimone (1985) positioned two stimuli in the receptive field of a V4 neuron and directed the animal's attention to one of the stimuli. The neural responses were consistent with the idea that attention caused the receptive field to shrink around the attended area of space. This would alter receptive field profile in a way inconsistent with multiplicative scaling. Support for this hypothesis was provided by Connor et al. (1996, 1997), who measured the neuronal responses to a bar placed at different locations within the receptive field while directing the animal's attention to different areas adjacent to the receptive field. Their results suggested that the receptive field of the neuron shifted toward the attended location.

It remains to be seen whether this shift in the receptive field profile represents a different mode of action for attentional modulation. It is important to note that this effect could arise from a multiplicative scaling of attention of the receptive fields of cortical neurons in earlier stages of cortex. Receptive fields of cortical neurons increase in size in later stages of cortical processing, because of the convergence of inputs from preceding visual areas. Because attention can be localized to a specific spatial location (Posner, 1980; Eriksen and St. James, 1986), attention might only modulate responses of neurons whose receptive fields overlap that location. The site of action of attention might be earlier in visual cortex when a small domain is attended and later in cortex when a large domain is attended. But what happens if we record from a neuron whose receptive field size is greater than the size of the location to which attention was directed? That neuron will receive inputs from neurons whose responses were enhanced by attention, as well as inputs from neurons whose responses were not altered by attention because their receptive field did not contain the attended location. The experimenter will measure a shift in the receptive field profile toward the attended location because the inputs from the attended location will have been enhanced by attention relative to the inputs from the unattended location. Although the immediate effect of attention was a multiplicative scaling, the consequences at later stages would not be.
This hypothesis assumes that attention can selectively target either cells at earlier stages of visual processing with smaller receptive fields or target the inputs to a neuron that arrive from different spatial locations. Area V4 is relatively early in sensory processing, but inputs arriving from area V2 or area V1 may show some attentional modulations. Our experiments found that $31 \%$ of the V1 neurons were modulated by attention, and others have reported similar results (Motter, 1993; Press and Van Essen, 1997). A spatial shifting of V4 receptive fields may be caused by the asymmetry that arises in the inputs to the V4 cells, when some of those inputs cover an enhanced or attended location, and others arise from neighboring unattended regions.

The current experiments have shown that attention causes a multiplicative scaling of orientation-tuning functions in area V4. Because multiplicative scaling is also seen in the interactions of many stimulus dimensions with each other, it is likely to represent an important aspect of cortical signaling. It will be important to determine whether the result is general to other tasks, stimulus dimensions, and areas. If extraretinal and retinal inputs routinely use similar mechanisms to modulate neuronal responses, characterizing the integration of behavior and sensory signals might be greatly simplified.

\section{REFERENCES}

Adelson EH, Bergen JR (1985) Spatiotemporal energy models for the perception of motion. J Opt Soc Am 2:284-299.

Albrecht DG, Geisler WS (1991) Motion selectivity and the contrastresponse function of simple cells in the visual cortex. Vis Neurosci 7:531-546.

Albrecht DG, Hamilton DB (1982) Striate cortex of monkey and cat: contrast response function. J Neurophysiol 48:217-237.

Andersen RA, Essick GK, Siegel R (1985) The encoding of spatial location by posterior parietal neurons. Science 230:456-458.

Bisti S, Carmignoto G, Galli L, Maffei L (1985) Spatial-frequency characteristics of neurones of area 18 in the cat: dependence on the velocity of the visual stimulus. J Physiol (Lond) 359:259-268.

Bricolo E, Ipata AE, Chelazzi L, Treves A (1997) Slow build-up of color and orientation selectivity in area V4. Soc Neurosci Abstr 23:2064.

Carandini M, Heeger DJ (1994) Summation and division by neurons in primate visual cortex. Science 264:1333-11336.

Connor CE, Gallant JL, Preddie DC, Van Essen DC (1996) Responses in area V4 depend on the spatial relationship between stimulus and attention. J Neurophysiol 75:1306-1308.

Connor CE, Preddie DC, Gallant JL, Van Essen DC (1997) Spatial attention effects in macaque area V4. J Neurosci 17:3201-3214.

De Valois RL, Albrecht DG, Thorell LG (1982) Spatial frequency selectivity of cells in macaque visual cortex. Vision Res 22:545-559.

Dean AF (1981) The variability of discharge of simple cells in the cat striate cortex. Exp Brain Res 44:437-440.

Desimone R, Duncan J (1995) Neural mechanisms of selective visual attention. Annu Rev Neurosci 18:193-222.

Eriksen CW, St. James JD (1986) Visual attention within and around the field of focal attention: a zoom lens model. Percept Psychophys 40:225-240.

Foster KH, Gaska JP, Nagler M, Pollen DA (1985) Spatial and temporal frequency selectivity of neurones in visual cortical areas V1 and V2 of the macaque monkey. J Physiol (Lond) 365:331-363.

Friend SM, Baker Jr CL (1993) Spatio-temporal frequency separability in area 18 neurons of the cat. Vision Res 33:1765-1771.

Galli L, Chalupa L, Maffei L, Bisti S (1988) The organization of receptive fields in area 18 neurones of the cat varies with the spatio-temporal characteristics of the visual stimulus. Exp Brain Res 71:1-7.

Geisler WS, Albrecht DG (1997) Visual cortex neurons in monkeys and cats: detection, discrimination and identification. Vis Neurosci 14:897-919.

Haenny PE, Schiller PH (1988) State dependent activity in monkey visual cortex. I. Single cell activity in V1 and V4 on visual tasks. Exp Brain Res 69:225-244.

Hamilton DB, Albrecht DG, Geisler WS (1989) Visual cortical receptive 
fields in monkey and cat: spatial and temporal phase transfer function. Vision Res 20:1285-1308.

Heggelund P, Albus K (1978) Response variability and orientation discrimination of single cells in striate cortex of cat. Exp Brain Res 32:197-211.

Henry GH, Bishop PO, Tupper RM, Dreher B (1973) Orientation specificity and response variability of cells in the striate cortex. Vision Res 13:1771-1779.

Holub RA, Morton-Gibson M (1981) Response of visual cortical neurons of the cat to moving sinusoidal gratings: response-contrast functions and spatiotemporal interactions. J Neurophysiol 46:1244-1259.

Humphreys G, Bruce V (1989) Visual cognition. London: Lawrence Erlbaum.

Ikeda H, Wright MJ (1972) Receptive field organisation of "sustained" and "transient" retinal ganglion cells which subserve different functional roles. J Physiol (Lond) 227:769-800.

Judge SJ, Richmond BJ, Chu FC (1980) Implantation of magnetic search coils for measurement of eye position: an improved method. Vision Res 20:535-538.

Luck SJ, Chelazzi L, Hillyard SA, Desimone R (1997) Neural mechanisms of spatial selective attention in areas V1, V2, and V4 of macaque visual cortex. J Neurophysiol 77:24-42.

Makous JC, Preddie DC, Connor CE, Van Essen DC (1997) Suppression and facilitation mediate spatial effects of directed attention in macaque area V4. Soc Neurosci Abstr 23:2064.

Maunsell JHR (1995) The brain's visual world: representations of visual targets in cerebral cortex. Science 270:764-769.

Maunsell JHR, Hochstein S (1991) Effects of behavioral state on the stimulus selectivity of neurons in area V4 of the macaque monkey. In: Channels in the visual nervous system: neurophysiology, psychophysics and models, (Blum B, ed), pp 447-470. London: Freund.

Maunsell JHR, Sclar G, Nealey TA, DePriest DD (1991) Extraretinal representations in area $\mathrm{V} 4$ in the macaque monkey. J Neurosci 7:561-573.

McLean J, Palmer LA (1994a) Contribution of linear mechanisms to the specification of local motion by simple cells in areas 17 and 19 of the cat. Vis Neurosci 11:271-294.

McLean J, Palmer LA (1994b) Organization of simple cell responses in the three-dimensional (3-D) frequency domain. Vis Neurosci 11:295-306.

Moran J, Desimone R (1985) Selective attention gates visual processing in the extrastriate cortex. Science 229:782-784.

Motter BC (1993) Focal attention produces spatially selective processing in visual cortical areas V1, V2, and V4 in the presence of competing stimuli. J Neurophysiol 70:909-919.

Motter BC (1994a) Neural correlates of attentive selection for color or luminance in extrastriate area V4. J Neurosci 14:2178-2189.
Motter BC (1994b) Neural correlates of feature selective memory and pop-out in extrastriate area V4. J Neurosci 14:2190-2199.

Parker A, Hawken M (1985) Capabilities of monkey cortical cells in spatial-resolution tasks. J Opt Soc Amer 2:1101-1114.

Posner MI (1980) Orienting of attention. Q J Exp Psychol 32:3-25.

Posner MI, Snyder CRR, Davidson BJ (1980) Attention and the detection of signals. J Exp Psychol Gen 109:160-174.

Preddie DC, Connor CE, Gallant JL, Van Essen DC (1995) Time course of attentional effects in macaque area V4. Soc Neurosci Abstr 21:1759.

Press WA, Van Essen DC (1997) Attentional modulation of neuronal responses in macaque area V1. Soc Neurosci Abstr 23:1026.

Press WH, Flanner BP, Teukolsky SA, Vetterling WT (1989) Numerical recipes in C. Cambridge: Cambridge UP.

Robinson DA (1963) A method of measuring eye movements using a scleral search coil in a magnetic field. IEEE Trans Biomed Eng 101:131-145.

Rossi AF, Paradiso MA (1995) Feature-specific effects of selective visual attention. Vision Res 35:621-634.

Schiller PH, Finlay BL, Volman SF (1976) Short-term response variability of monkey striate neurons. Brain Res 105:347-349.

Sclar G, Freeman RD (1982) Orientation selectivity in the cat's striate cortex is invariant with stimulus contrast. Exp Brain Res 46:457-461.

Shapley R, Victor J (1986) Hyperacuity in cat retinal ganglion cells. Science 231:999-1002.

Skottun BC, Ohzawa I, Sclar G, Freeman RD (1987) The effects of contrast on visual orientation and spatial frequency discrimination: a comparison of single cells and behavior. J Neurophysiol 57:773-786.

Spitzer H, Richmond BJ (1991) Task difficulty: ignoring, attending to, and discriminating a visual stimulus yield progressively more activity in inferior temporal neurons. Exp Brain Res 83:340-348.

Spitzer H, Desimone R, Moran J (1988) Increased attention enhances both behavioral and neuronal performance. Science 240:338-340.

Tolhurst DJ (1973) Separate channels for the analysis of the shape and the movement of a moving visual stimulus. J Physiol (Lond) 231:385-402.

Tolhurst DJ, Movshon JA (1975) Spatial and temporal contrast sensitivity of striate cortical neurons. Nature 257:674-675.

Treue S, Maunsell JHR (1996) Attentional modulation of visual motion processing in cortical areas MT and MST. Nature 382:539-541.

Vogels R, Orban GA (1990) How well do response changes of striate neurons signal differences in orientation: a study of the discriminating monkey. J Neurosci 10:3543-3558.

Vogels R, Orban GA (1994) Activity of inferior temporal neurons during orientation discrimination with successively presented gratings. J Neurophysiol 71:1428-1451.

Wolbarsht MI, MacNichol EF, Wagner HG (1960) Glass insulated platinum microelectrode. Science 132:1309-1310. 